\title{
A DISCRETE EVENT SIMULATION (DES) BASED APPROACH TO MAXIMIZE THE PATIENT THROUGHPUT IN OUTPATIENT CLINIC
}

\author{
Vidanelage L. Dayarathna ${ }^{1}$, Hebah Mimesh ${ }^{2}$, Mohammad Nagahisarchoghaei $^{3}$, Aziz Alhumoud ${ }^{4}$ \\ ${ }^{1} \mathrm{PhD}$ Student, Graduate Research Assistant, Department of Industrial and Systems Engineering, \\ Mississippi State University, PO Box 9542, Mississippi State, 39762, USA \\ ${ }^{2}$ Master Student, Department of Industrial and Systems Engineering, Mississippi State University, PO \\ Box 9542, Mississippi State, 39762, USA \\ ${ }^{3} \mathrm{PhD}$ Student, Graduate Research Assistant, College of Computing and Informatics, \\ University of North Carolina at Charlotte, North Carolina, USA \\ ${ }^{4}$ Department of Industrial and Systems Engineering, Mississippi State University, PO Box 9542, \\ Mississippi State, 39762, USA.
}

*Corresponding Author: Mohammad Nagahisarchoghaei

Corresponding Author Email: mn852@msstate.edu

Article Received: 15-04-19

Accepted: 15-07-19

Published: 05-08-19

Licensing Details: Author retains the right of this article. The article is distributed under the terms of the $\begin{array}{lllll}\text { Creative } & \text { Commons } & \text { Attribution-NonCommercial } & 4.0 & \text { License }\end{array}$ (http://www.creativecommons.org/licences/by-nc/4.0/) which permits non-commercial use, reproduction and distribution of the work without further permission provided the original work is attributed as specified on the Journal open access page

\begin{abstract}
The healthcare system is a complex system which exhibits conditions of uncertainty, ambiguity emergence that incurs incoming patient congestion. Discrete event simulation (FlexSim) is considered as a viable decision support tool in analyzing a system for improvement. Using a data-driven discrete event simulation approach, this paper portrays a comprehensive analysis to maximize the number of patients in an on-campus clinic, located at Mississippi State University. The outcome of the analysis of current system exhibits that deploying a few nurse practitioners results in bottlenecks which decreases the systems' throughput substantially due to the overall longer patients' waiting time. Access to the laboratory is characterized through multi-server queuing network, arrival process is followed discrete distributions, and batch sizes and arrival times are stochastic in nature. In an effort to plummet inpatient congestion at the outpatient clinic, by using empirically calibrated simulation model, we will figure out the best balance
\end{abstract}


between the number of the lab technician and incoming patient during working hour. An analysis of optimal solutions is demonstrated, which is followed by recommendation and avenues for future research.

Keywords: Healthcare, System Simulation, Discrete Event Simulation (DES), Patient Throughput, Waiting Time.

\section{INTRODUCTION AND PROBLEM DESCRIPTION}

Because of the increasing number of students and faculty, on-campus health centers are some of the most demanding outpatient clinics. Campus health centers are a convenient, and usually affordable, location for college students to obtain quick, effective health care service, so they can maintain an active academic schedule. On-campus Mississippi State University health center is under increasing pressure to reduce waste, eliminate unnecessary costs and redundant efforts, thereby improving the quality and consistency of healthcare delivery. Lack of automation is a critical factor that can help improving process efficiency. Recently, many research has been conducted related to healthcare simulation. Various models and techniques have been developed and analyzed to determine the optimal solution of various healthcare system problems. De Vasconcelos et al. (2018), Moon et al. (2015), Prodel et al. (2014), Nagahisarchoghaei et al. (2018), Abutabenjeh et al. (2019), Ahmadi et al. (2014a, 2014b), Raunak et al. (2009), Hossain et al. (2017) used simulation techniques in emergency healthcare systems. Chuang et al. (2018), Roh et al. (2013), Marmor et al. (2013), Soleimani et al. (2018) Nagahi et al. (2018), Hossain and Jaradat (2018), and Tyler et al. (2003) developed other models to provide better resource management solutions for the healthcare sector, and Kabaso et al. (2015), Montañola-Sales et al. (2015), and Adams et al. (1998) developed varied simulation models to circumvent the severity of pandemic diseases. Chen et al. (2018), Norouzzadeh et al. (2015), Jin et al. (2013), and Jacobson et al. (2006) focused on improving the healthcare system's performance by dint of reducing patient waiting time. Monahan and Fabbri (2018), Turkcan et al. (2014), Hossain et al. (2019), and Kaandorp and Koole (2007) concentrate on improving appointment scheduling techniques. Other than the healthcare simulation models, Ma et al. (2019) have developed a Virtual Reality based teaching module to provide more immersive experience to the users in a simulated way.

Because of the dynamic and emergent nature of healthcare systems, the patient wait time is still a big issue in today's healthcare systems. Thus there is an need to employ a "system approach" to better handle the intricacies stemming from any kind of complex system (Alfaqiri, 2019; Hossain \& Jaradat, 2016, 2018; Lawrence et al., 2019; Nagahi, Hossain, \& Jaradat, 2019; Nagahi \& Jaradat, 2019; Nagahi et al., 2019; Stirgus et al., 2019).Therefore, we used a discrete event simulation -FlexSim to find optimal ways to reduce patient wait time in this paper and select the John C. Longest Student Health Center (LSHC) at Msstate as a case study to reduce patient wait time for the X-ray laboratory. LSHC employs physicians and nurses who are qualified to treat patients from most illnesses and capable of handling most medical emergencies including x-rays, shots, blood work, and trauma (UHS, 2019). The health center hours are from 8 am to 5 pm 
during the week, and during those hours the health center is capable of assisting up to 200 patients. Most patients are treated adequately with a short amount of time. However, patients in need of X-rays usually need more time to go through the process of treatment. Only one technician handle the X-ray work process (taking picture) which takes around 5-15 minutes per patient. Our objective is to reduce bottlenecks that are created in the X-ray lab, and reduce patient's service time in the X-ray lab. By achieving this objective, more LSHC patients will be treated with a better quality of treatment. These objectives will be met by using Flexsim software to build a model and simulate the system under different variables that affect service utilization and work in process time.

In the current system, X-ray patients are routed in three ways, normal route and 2 reroute routes. The normal route requires patients to wait in the initial queue, enter the X-ray lab till technician take their picture, wait in a second queue to see the doctor (main processor), see the doctor and then exit the system (see Figure 1). The second and third reroute route requires patients to wait in the initial queue, enter the X-ray lab, see the subsidiary doctors (second and third processors) and then leave the system. The current system (LSHC) has $70 \%$ of patients routed through the normal route and $15 \%$ rerouted in second and third routs, respectively. We hypothesize that increasing the percentage of patients who are rerouted will decrease waiting times and increase the throughput of the entire system. Observations and data have been collected and inserted into the developed simulation model (see Figure 1). To show the real system, we developed a simulation model by using FlexSim software. According to the data collection, we manipulated and analyzed different percentages of X-ray patients in each route to obtain an optimal solution for patient routing, to reduce wait time, and to increase system throughput without actual changes in the current system.

The objectives of the paper are:

- To evaluate why bottlenecks are created in the X-ray Lab.

- To come up with potential recommendations to reduce patient's waiting time in the X-ray Lab.

- To observe how manipulating patient routing might impact the wait time and throughput of the system in the X-ray lab.

Following the introduction, the second section illustrates the developed simulation model in detail, and the third section shows the experimental design analysis, and the fourth section validates the proposed simulation model. The fifth section presents an analysis of the model output. Finally, the last section gives some recommendations based on the analysis results.

\section{SIMULATION MODEL}

To propose a simulation model for the study, this section is divided to four parts including, modeling assumption, key performance measures, key decision variables, and system description.

\section{Modeling Assumptions}

Following assumptions are considered to create the simulated model: 
(1) All patients entering the system will necessitate x-ray work. No other patient type will be considered in the system. Patients will only be able to enter the system from $8 \mathrm{pm}-4 \mathrm{pm}$.

(2) The first patient to enter the system will be the first patient to exit the system

(3) The system will operate for nine consecutive hours to represent the normal operation hours of 8 am to $5 \mathrm{pm}$. However, the source will close one hour before the rest of the system to allow all patients to travel the length of the system (The actual system does not always abide by this and fluctuates its closing hours. This will likely impact the throughput of the system which will be explored in the validation results.)

(4) Physicians, nurses, and technicians will always be available when the processors are empty of previous patients.

(5) There will be no bulking or reneging.

(6) Arrival rate will follow a Triangular Distribution (because of lack of data, the distribution is not certain) and service time will be independent (exponential distribution).

(7) For the Qu_X-Ray and the Qu_Doctor1 have a maximum capacity of 5 and 3 patients respectively.

\section{Key Performance Measure}

The performance measures of the system are presented as follows:

- The average wait time of patients in the second Queue (Qu-report)

- The throughput of the X-ray lab (entire system).

\section{Key Decision Variables}

The decision variable of the system is the percentage of patients that move through the normal route and two rerouted routes. Currently, the system flows $70 \%$ of the patients through the normal route and $30 \%$ of patients through the rerouted routes. Other than X-ray patients, no other patients entered the system for any case. The time that the system open for patients is from 8 a.m. to 4 p.m.

\section{System Description}

The simulation model presented is initiated by the arrival of patients in the system. Patients are then required to wait in the $\mathrm{X}$-ray queue labeled $\mathrm{Qu}$ _X_ray in figure 1. Patients would wait to be processed in the X-ray room in order to take X-rays as specified. Once patients have been processed they are sent to the normal route which is the Qu_report, and follows is the Pr_Doctor who is a doctor that explains the X-rays and results. The normal route is taken by $70 \%$ of patients. The other $30 \%$ of patients are divided equally by two other doctors (Pr_Doctor_2_Reroute and Pr_Doctor_3_Reroute). The 30\% are immediately seen by a doctor after they have been processed in the $\mathrm{X}$-ray room (Pr_X_ray) and then sent to the sink which is exiting the system. Using this system, we have eliminated two queues for doctor 2 and 3. Figure 
1 is a snapshot of the model using Flexsim, and Figure 2 is the object flow diagram (OFD) of the system. Both figures are presented below.

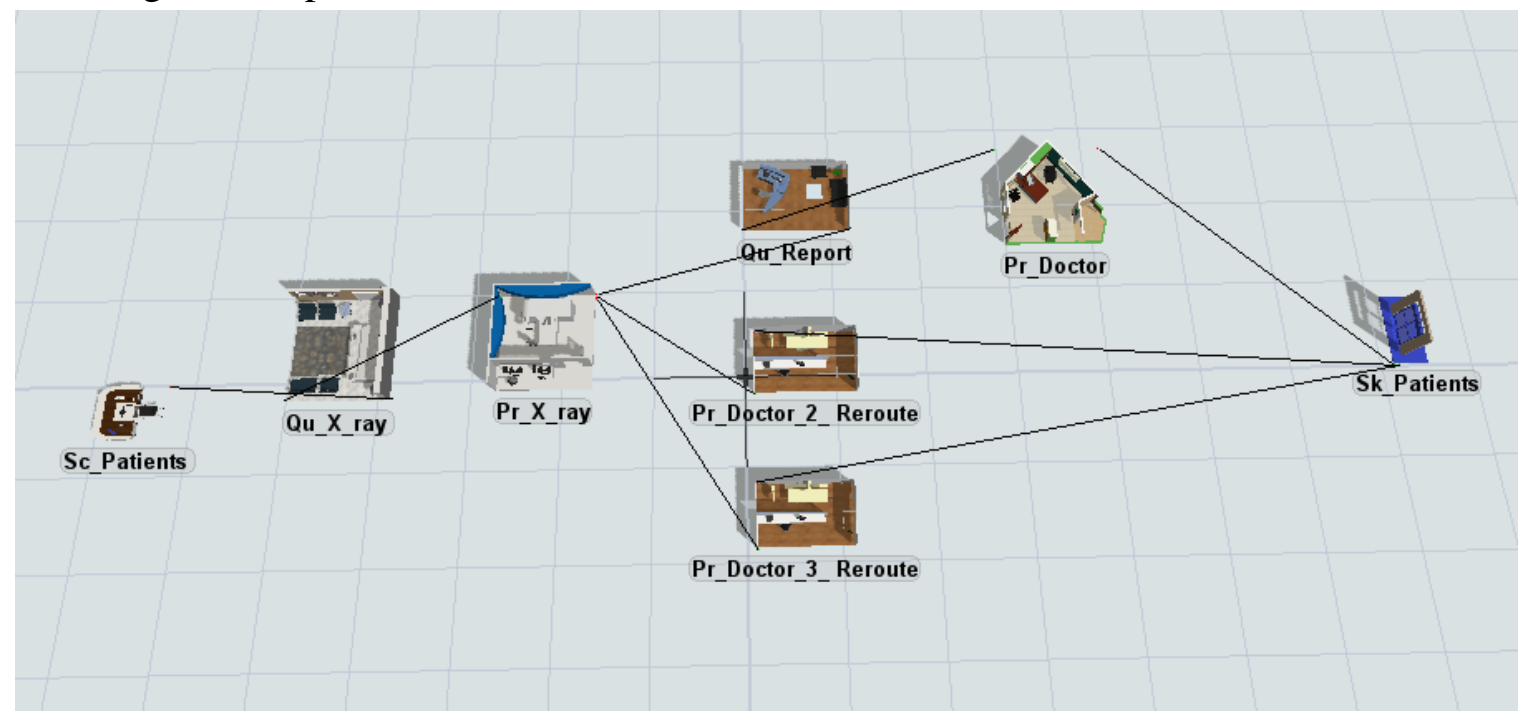

Figure 1. Model view in FlexSim interface

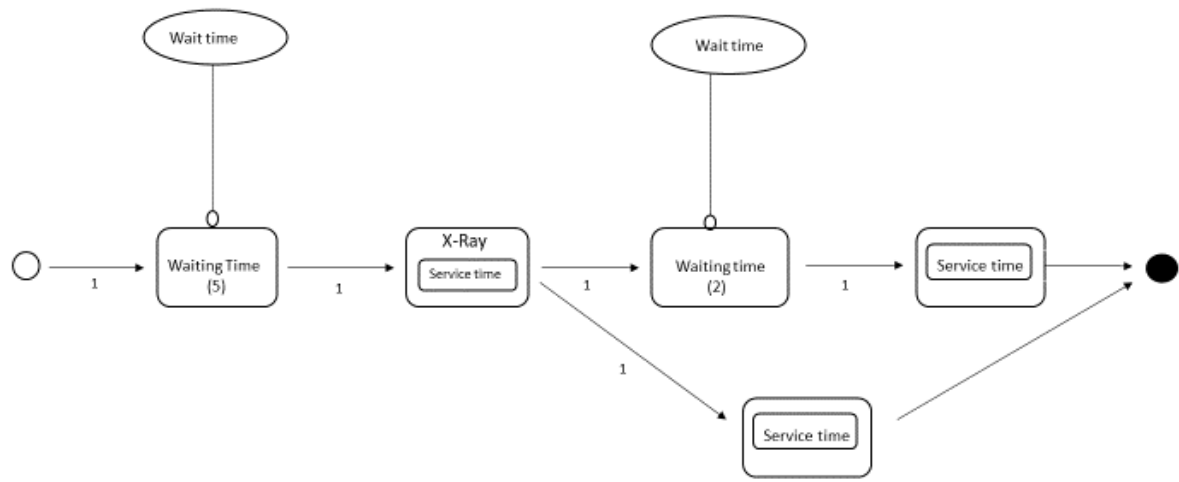

OFD of X-ray

Figure 2. Object flow diagram (OFD) of X-ray Laboratory

The Basic properties of fixed resources are as follows:

Table 1: Basic properties of fixed resources

\begin{tabular}{ccccc}
\hline Object name & Description & Capacity & Downtime & Process Time \\
\hline Sc_Patients & Source & --- & $4 \mathrm{pm}-9 \mathrm{am}$ & --- \\
Qu_X-ray & Queue for X-ray & 5 & $5 \mathrm{pm}-9 \mathrm{am}$ & --- \\
Pr_X-ray & X-ray processor & 1 & $5 \mathrm{pm}-9 \mathrm{am}$ & $4 \mathrm{~min}$ \\
Qu_Report & Queue for Doctor & 3 & $5 \mathrm{pm}-9 \mathrm{am}$ & --- \\
Pr_Doctor_1 & Doctor 1 processor & 1 & $5 \mathrm{pm}-9 \mathrm{am}$ & $15 \mathrm{~min}$ \\
Pr_Doctor_2_ & Doctor 2 processor & 1 & $5 \mathrm{pm}-9 \mathrm{am}$ & $15 \mathrm{~min}$ \\
\hline
\end{tabular}




\begin{tabular}{ccccc}
\hline Reroute & & & \\
Pr_Doctor_3_ & Doctor 3 reroute & 1 & $5 \mathrm{pm}-9 \mathrm{am}$ & $15 \mathrm{~min}$ \\
Reroute & processor & & & \\
Sk_Patients & Sink & --- & $5 \mathrm{pm}-9 \mathrm{am}$ & --- \\
\hline
\end{tabular}

\section{MODEL VALIDATION}

The Student health center provides services to approximately 20,000 patients in 9 month time period when the university is in session. The implemented model with initial condition results in 24,563 patients over the same period. The reason for the discrepancy is no doctor (processors) dedicated only for X-ray lab. Those three doctors examine patients with different kinds of issues from the cold, flu, X-ray, and so on. The second reason is that the changes in health center opening and closing time affect the operation hours.

\section{EXPERIMENTAL DESIGN}

The source (Sc_Patients) follows a Triangular (5, 7, and 6) distribution to uphold entering six patients per hour to the system. The capacity of the Queue (Qu_X-ray) is 5, and all patients wait there before receiving the service. A global table is created with initial conditions of $70 \%$ of Route I, $15 \%$ of Route II, and 15\% of Route III. Processor (Pr_X-ray) uses to send to port by case with Route I sent to Qu_Report (normal route), Route II sent to Pr_Doctor_2_Reroute (Doctor 2 reroute), and Route III sent to Pr_Doctor_3_ Reroute (Doctor 3 reroute). The model runs for 259,200 minutes. The time is based on 180 working days with 9 hours of each day over 9 month period. To account for variance between separate replications, the model is set to run ten replications. A warm-up period is not included as no patients are waiting in line at the beginning of each day. The experiments details are summarized in Table 2.

Table 2: Experiments details

\begin{tabular}{cccccc}
\hline & \multicolumn{3}{c}{ Variables } & \multicolumn{2}{c}{ Performance Measures } \\
\cline { 2 - 6 } & Global & Global & Global & & System \\
& Table Row & Table Row & Table Row & Qu_Report & Throughput \\
Scenario & 1/Col 1 & 2/Col 2 & 3/Col 3 & Wait Time min & $\begin{array}{c}\text { (Patients/9 } \\
\text { months) }\end{array}$ \\
1 & 70 & 15 & 15 & 43 & 24563 \\
2 & 80 & 10 & 10 & 45 & 21532 \\
3 & 90 & 5 & 5 & 45 & 19191 \\
4 & 60 & 20 & 20 & 40 & 28309 \\
5 & 50 & 25 & 25 & 34 & 32462 \\
6 & 40 & 30 & 30 & 22 & 34312 \\
\hline
\end{tabular}

Figure 3 represents the Qu_Report wait time for the six scenarios and Figure 4 shows the throughput of the system for the same scenarios. 


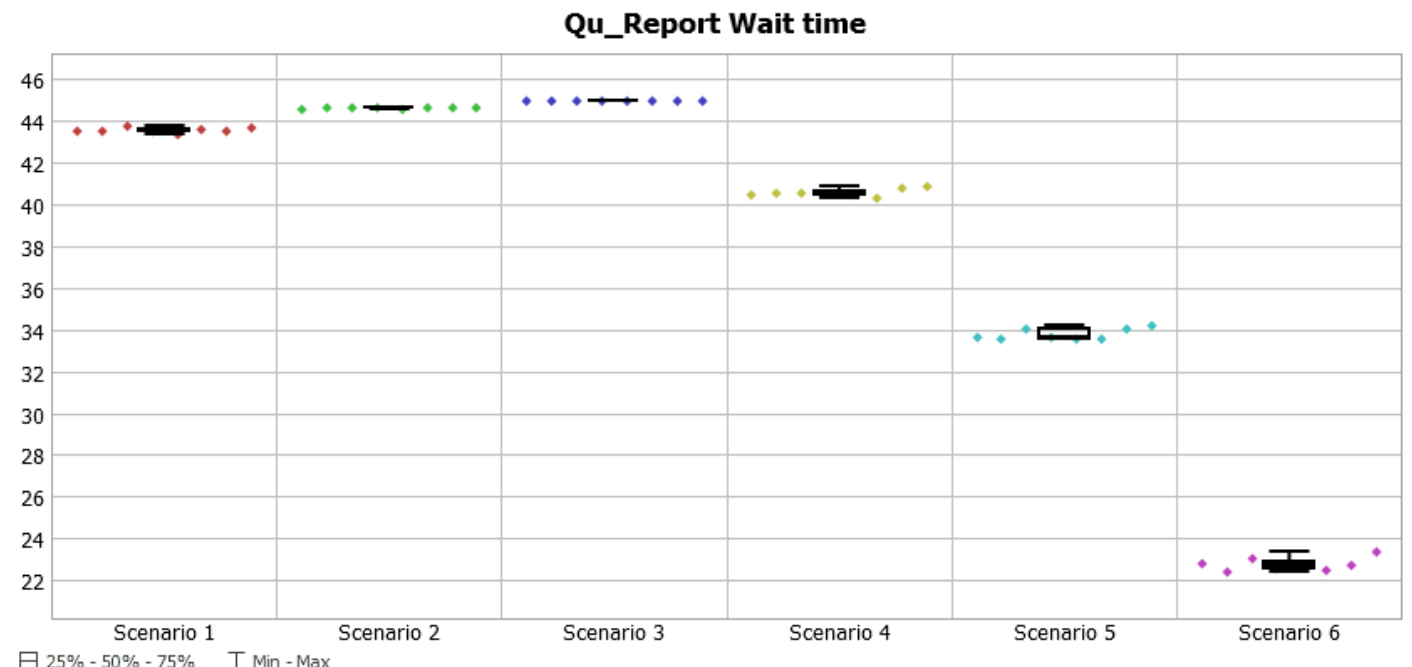

Figure 3. Qu_Report Wait time analysis under six scenarios

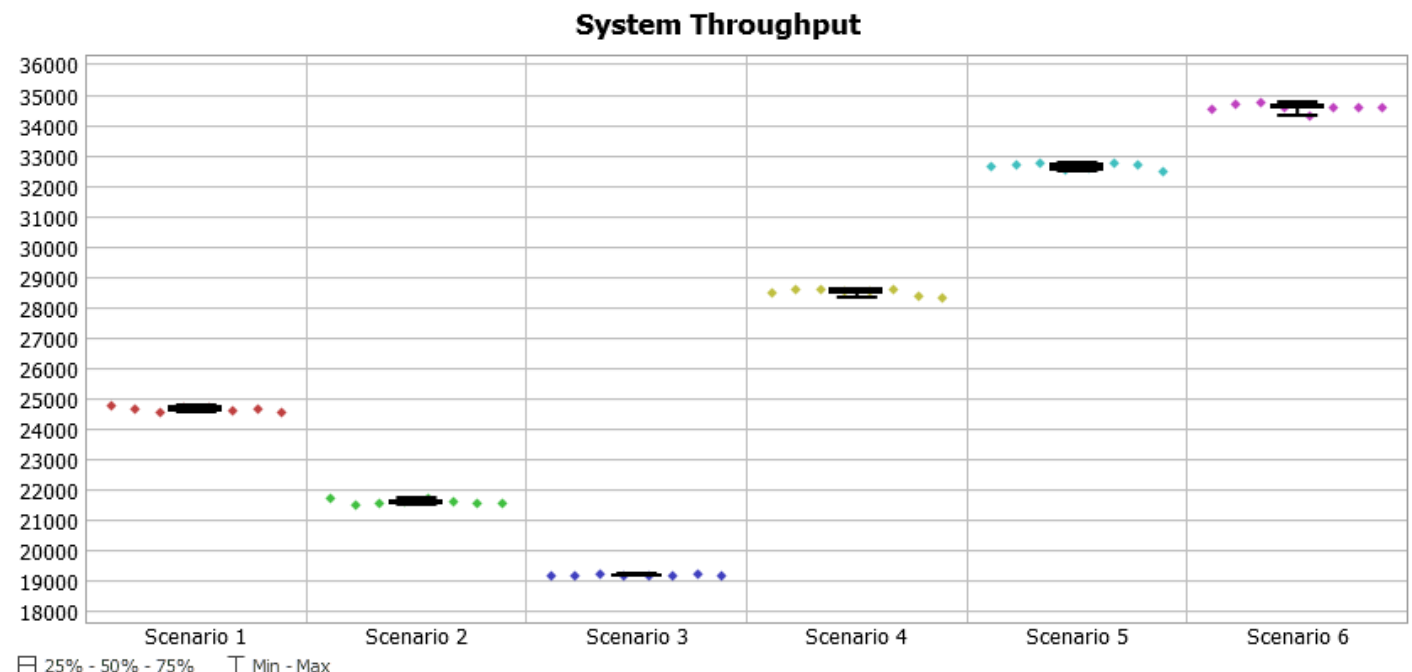

Figure 4. System throughput analysis under various scenarios

\section{RESULT OF ANALYSIS}

The system is designed to manipulate the actual X-ray lab system in LSHC as closely as possible with stated assumptions. A bottleneck is identified in the queue ( $Q u$ Report) through a system run with initial conditions. The global table is used to define the percentages of patients labeled Route I (Sc_Patients- Qu_X-ray- Pr_X-ray- Qu_Report- Pr_Doctor - Sk_Paitents), Route II (Sc_Patients- Qu_X-ray- Pr_X-ray- Pr_Doctor_2_Reroute- Sk_Patients), and Route III (Sc_Patients- Qu_X-ray-Pr_X-ray-Pr_Doctor_3_Reroute- Sk_Patients) and manipulate the percentages to check the normal route and reroute routes. In Flexim, the Experimenter option under the Statistics tab is used to analyze different scenarios. For each scenario, Qu_Report Wait Time and System Throughput were calculated to analyze the system. Based on the results above, 
a ratio of $40 \%$ Route I, $30 \%$ Route II, and 30\% route III showed the lowest mean wait time (22 min) in Qu_Report and greatest System Throughput (34312 patients/9months).

\section{CONCLUSIONS AND RECOMMENDATIONS}

The current system rerouting $30 \%$ of patients (15\% each) to Doctor 2 and Doctor 3 has created the bottleneck in the queue which results in higher waiting time. The results of this study suggest $40 \%$ of patients should follow the normal route in the x-ray lab, and $30 \%$ should be rerouted directly to Doctor 2, and the other $30 \%$ should be rerouted to Doctor 3 . The increasing percentages in reroutes decrease waiting time and increase the throughput of the system by $\sim 40 \%$. Based on the results, it is recommended that John C. Longest Student Health Center should increase the percentage of rerouting from $30 \%$ to $60 \%$.

\section{ACKNOWLEDGMENT}

Authors would like to express their appreciation to Dr. Raed Jaradat, assistant professor, and Niamat Ullah Ibne Hossain, doctoral student, from the Department of Industrial and Systems Engineering at Mississippi State University, for their constructive guidance.

\section{References}

Abutabenjeh, S., Dayarathna, L., Nagahi, M., Jaradat, R., \& Gordon, S., (2019). The perceived value of public procurement and contract management certification. International Journal of Procurement Management, 11(3) (in press).

Adams, A. L., Barth-Jones, D. C., Chick, S. E., \& Koopman, J. S. (1998). Simulations to evaluate HIV vaccine trial designs. Simulation, 71(4), 228-241.

Ahmadi, S. A. A., Tajabadi, S. H., Nagahi, M., \& Sarchoghaei, M. N. (2014a). Effect of LeaderMember Exchange on Perceived Organizational Support. International Journal of Research in Organizational Behaviour and Human Resource Management, 2(1), 98.

Ahmadi, S. A. A., Azar, H. K., Sarchoghaei, M. N., \& Nagahi, M. (2014b). Relationship between Emotional Intelligence and Psychological Well Being. International Journal of Research in Organizational Behavior and Human Resource Management, 2(1), 123-144.

Alfaqiri, A., Hossain, N. U., Jaradat, R., Abutabenjeh, S., Keating, C., Khasawneh, M., \& Pinto, A. (2019). A systemic approach for disruption risk assessment in oil and gas supply chains. International Journal of Critical Infrastructures, 15(3).

Chen, N., Wang, M., Xie, X., Zheng, L., \& Swartz, C. H. (2018). Modeling and Analysis of the Waiting Time of Rapid Response Process in Acute Care. IEEE Robotics and Automation Letters, 3(1), 336-343.

Chuang, M. T., Hu, Y. H., \& Lo, C. L. (2018). Predicting the prolonged length of stay of general surgery patients: a supervised learning approach. International Transactions in Operational Research, 25(1), 75-90.

De Vasconcelos Silva, A. C. P., Assad, D. B. N., \& Spiegel, T. (2018). Capacity Decision in Emergency Hospital Operation Rooms: Sizing Using Simulation. In Modeling and Simulation Techniques for Improved Business Processes (pp. 140-155). IGI Global.

Hossain, N. U. I. (2018). Leveraging six sigma approach to reduce clinic patient waiting time. 
In Proceedings of the International Annual Conference of the American Society for Engineering Management. (pp. 1-6). American Society for Engineering Management (ASEM).

Hossain, N. U. I. \& Jaradat, R. (2018). A synthesis of definitions for systems engineering. In Proceedings of the International Annual Conference of the American Society for Engineering Management. (pp. 1-10). American Society for Engineering Management (ASEM).

Hossain, N. U. I., Debusk, H., \& Hasan, M. M. (2017). Reducing patient waiting time in an outpatient clinic: a discrete event simulation (DES) based approach. In IIE Annual Conference. Proceedings (pp. 241-246). Institute of Industrial and Systems Engineers (IISE).

Hossain, N. U. I., Nur, F., \& Jaradat, R. M. (2016). An analytical study of hazards and risk in the shipbuilding study. Proceedings of the International Annual Conference of the American Society for Engineering Management. (pp. 1-8). American Society for Engineering Management (ASEM).

Hossain, N. U. I., Nagahi, M., Jaradat, R., \& Keating, C. (2019) Development of an Instrument to Assess the Performance of Systems Engineers. In Proceedings of the International Conference on Industrial Engineering and Operations Management, Toronto, Canada, October 23-25, 2019 (in press).

Jacobson, S. H., Hall, S. N., \& Swisher, J. R. (2006). Discrete-event simulation of health care systems. In Patient flow: Reducing delay in healthcare delivery (pp. 211-252). Springer, Boston, MA.

Jin, X., Sivakumar, A. I., \& Lim, S. Y. (2013, December). A simulation based analysis on reducing patient waiting time for consultation in an outpatient eye clinic. In Proceedings of the 2013 Winter Simulation Conference: Simulation: Making Decisions in a Complex World (pp. 2192-2203). IEEE Press.

Kaandorp, G. C., \& Koole, G. (2007). Optimal outpatient appointment scheduling. Health Care Management Science, 10(3), 217-229.

Kabaso, E. M., Currie, C. S., \& Brailsford, S. C. (2015, December). Simulating the provision of antiretroviral therapy in Zambia. In 2015 Winter Simulation Conference (WSC) (pp. 1285-1294). IEEE

Lawrence, JM., Hossain, N. U. I., Nagahi, M., \& Jaradat, R. (2019). Impact of a Cloud-Based Applied Supply Chain Network Simulation Tool on Developing Systems Thinking Skills of Undergraduate Students. In Proceedings of the International Conference on Industrial Engineering and Operations Management, Toronto, Canada, October 23-25, 2019 (in press).

Ma, J., Jaradat, R., Ashour, O., Hamilton, M., Jones, P., \& Dayarathna, V. L. (2019). Efficacy Investigation of Virtual Reality Teaching Module in Manufacturing System Design Course. Journal of Mechanical Design, 141(1), 012002. 
Marmor, Y. N., Rohleder, T. R., Cook, D. J., Huschka, T. R., \& Thompson, J. E. (2013). Recovery bed planning in cardiovascular surgery: a simulation case study. Health care management science, 16(4), 314-327.

Moon, I. C., Bae, J. W., Lee, J., Kim, D., Lee, H., Lee, T., ... \& Kim, G. W. (2015, December). EMSSIM: emergency medical service simulator with geographic and medical details. In Proceedings of the 2015 Winter Simulation Conference (pp. 1272-1284). IEEE Press.

Montañola-Sales, C., Gilabert-Navarro, J. F., Casanovas-Garcia, J., Prats, C., López, D., Valls, J., .. \& Vilaplana, C. (2015, December). Modeling tuberculosis in Barcelona: a solution to speed-up agent-based simulations. In Proceedings of the 2015 Winter Simulation Conference (pp. 1295-1306). IEEE Press.

Monahan, K., \& Fabbri, D. (2018). Schedule-based metrics for the evaluation of clinic performance and potential recovery of cancelled appointments. International journal of medical informatics, 109, 49-54.

Nagahi, M., Nagahisarchoghaei, M., Soleimani, N., \& Jaradat, R. M., (2018). Hedge strategies of corporate houses. Journal of Business Administration Research,7(1), 6-21.

Nagahi, M., Hossain, N. U. I., \& Jaradat, R. (2019). Gender differences in practitioners' preferences for systems-thinking skills. In proceeding of American Society for Engineering Management 2019 International Annual Conference and 40th Annual Meeting (in press).

Nagahi, M., Hossain, N. U. I., Jaradat, R., \& Grogan, S. (2019). Moderation Effect of Managerial Experience on the Level of Systems-Thinking Skills. In proceeding of the 13th Annual IEEE International Systems Conference. Available at SSRN 3363195.

Nagahi, M. \& Jaradat, R. (2019). The Impact of Practitioners' Personality Types on Their Level of Systems-Thinking Skills Preferences. Engineering Management journal, 31(3).

Nagahisarchoghaei, M., Nagahi, M., \& Soleimani, N. (2018). Impact of Exchange Rate Movements on Indian Firm Performance. International Journal of Finance and Accounting, 7(4), 108-121.

Norouzzadeh, S., Riebling, N., Carter, L., Conigliaro, J., \& Doerfler, M. E. (2015, December). Simulation modeling to optimize healthcare delivery in an outpatient clinic. In 2015 Winter Simulation Conference (WSC) (pp. 1355-1366). IEEE.

Prodel, M., Augusto, V., \& Xie, X. (2014, December). Hospitalization admission control of emergency patients using markovian decision processes and discrete event simulation. In Proceedings of the 2014 Winter Simulation Conference (pp. 1433-1444). IEEE Press.

Raunak, M., Osterweil, L., Wise, A., Clarke, L., \& Henneman, P. (2009, May). Simulating patient flow through an emergency department using process-driven discrete event simulation. In Proceedings of the 2009 ICSE Workshop on Software Engineering in Health Care (pp. 73-83). IEEE Computer Society.

Roh, T. P., Huschka, T. R., Brown, M. J., \& Marmor, Y. N. (2014, December). Data-driven simulation use to determine bed resource requirements for the redesign of pre-and post-

Engineering Science \& Technology Journal, Dayarathna, Mimesh, Nagahisarchoghaei, \& Alhumoud, PP 1-11 Page 10 
operative care areas. In Proceedings of the Winter Simulation Conference 2014 (pp. 1168-1176). IEEE.

Soleimani, N., Nagahi, M., Nagahisarchoghaei, M., \& Jaradat, R. (2018). The Relationship between Personality Types and the Cognitive-Metacognitive Strategies. Journal of Studies in Education, 8(2), 29-44.

Stirgus, E., Nagahi, M., Ma, J., Jaradat, R., Strawderman, L., \& Eakin, D., (2019) Determinants of systems thinking in college engineering students: research initiation. In proceeding of the 126th Annual Conference \& Exposition American Society for Engineering Education (in press).

Turkcan, A., Toscos, T., \& Doebbeling, B. N. (2014). Patient-centered appointment scheduling using agent-based simulation. In AMIA Annual Symposium Proceedings (Vol. 2014, p. 1125). American Medical Informatics Association.

Tyler, D. C., Pasquariello, C. A., \& Chen, C. H. (2003). Determining optimum operating room utilization. Anesthesia \& Analgesia, 96(4), 1114-1121.

University Health Service (UHS) at Mississippi State University. Retrieve from: https://www.health.msstate.edu/ 